\title{
A New Approach for Solving the Undamped Helmholtz Oscillator for the Given Arbitrary Initial Conditions and Its Physical Applications
}

\author{
Alvaro H. Salas S $\mathbb{D}^{1},{ }^{1}$ Jairo E. Castillo $H,{ }^{2}$ and Darin J. Mosquera $\mathbf{P}^{3}$ \\ ${ }^{1}$ Universidad Nacional de Colombia, Department of Mathematics and Statistics, FIZMAKO Research Group, Bogota, Colombia \\ ${ }^{2}$ Universidad Distrital Francisco José de Caldas, FIZMAKO Research Group, Bogota, Colombia \\ ${ }^{3}$ Universidad Distrital Francisco José de Caldas, ORION Research Group, Bogota, Colombia
}

Correspondence should be addressed to Alvaro H. Salas S; ahsalass@unal.edu.co

Received 2 May 2020; Revised 17 July 2020; Accepted 7 August 2020; Published 7 October 2020

Academic Editor: Francesco Clementi

Copyright (c) 2020 Alvaro H. Salas S et al. This is an open access article distributed under the Creative Commons Attribution License, which permits unrestricted use, distribution, and reproduction in any medium, provided the original work is properly cited.

\begin{abstract}
In this paper, a new analytical solution to the undamped Helmholtz oscillator equation in terms of the Weierstrass elliptic function is reported. The solution is given for any arbitrary initial conditions. A comparison between our new solution and the numerical approximate solution using the Range Kutta approach is performed. We think that the methodology employed here may be useful in the study of several nonlinear problems described by a differential equation of the form $z^{\prime \prime}=F(z)$ in the sense that $z=z(t)$. In this context, our solutions are applied to some physical applications such as the signal that can propagate in the LC series circuits. Also, these solutions were used to describe and investigate some oscillations in plasma physics such as oscillations in electronegative plasma with Maxwellian electrons and negative ions.
\end{abstract}

\section{Introduction}

Early descriptions of many of them date back to, at least, 1892, when the book by Greenhill [1] appeared, presenting a variety of such problems: a simple pendulum, catenaries, and a uniform chain that rotates steadily with a constant angular velocity about an axis to which the chain is fixed at two points. Later applications include nonlinear plasma oscillations [2], Duffing oscillators [3], rigid plates satisfying the Johansen yield criterion [4], nonlinear transverse vibrations of a plate carrying a concentrated mass [5], a beam supported at an axially oscillating mount [6], doubly periodic cracks subjected to concentrated forces [7], surface waves in a plasma column [8], coupled modes of nonlinear flexural vibrations of a circular ring [9], dualspin spacecrafts [10], spacecraft motion about slowly rotating asteroids [11], nonlinear vibration of buckled beams [12], a nonlinear wave equation [13], deep-water waves with two-dimensional surface patterns [14], oscillations of a body with an orbital tethered system [15], and nonlinear mathematical models of DNA [16, 17]. Numerical studies of phase spaces, stability analysis, solution by means of finite differences, application of the Bernoulli wavelet method for estimating a solution of linear stochastic integral equations, existence of periodic solutions, and numerical simulations can be found in [18-22]. The search for new analytical methods that lead to the exact solution of the Helmholtz equations is the vital importance, since the developed methods can be applied to Schrodinger's nonlinear differential equation, which is known to have different applications in nonlinear optics, plasma physics, fluid mechanics, and Bose-Einstein condensates $[23,24]$. As a contribution to the literature, in this article, we present the exact solution to the Helmholtz Oscillator for the given arbitrary initial conditions by means of the Weierstrass elliptic function.

In this paper, we will derive the exact solution to the Helmholtz oscillator: 


$$
\begin{aligned}
\ddot{q}+\alpha+\beta q+\gamma q^{2} & =0 \\
q(0) & =q_{0} \\
q^{\prime}(0) & =\dot{q}_{0}
\end{aligned}
$$

where $q \equiv q(t)$ denotes the displacement of the system, $\left.q^{\prime}(0) \equiv \partial_{t} q\right|_{t=0} \equiv \dot{q}_{0}, \ddot{q} \equiv \partial_{t}^{2} q, \beta$ is the natural frequency, $\gamma$ is a nonlinear system parameter, and $\alpha$ is a system parameter independent of the time.

Equation (1) is applied for mathematical modeling in physics and engineering like general relativity, betatron oscillations, vibrations of shells, vibrations of the acoustically driven human eardrum, and solid-state physics [24-28]. The Helmholtz oscillator can be interpreted as a particle moving in a quadratic potential field, and it has also been studied in a nonlinear circuit theory.

One of the possible interesting interpretations of equation (1) is given by a simple electrical circuit.

\section{The Physical Models and Helmholtz Equation}

2.1. Alternation the LC Series Circuit. Let us consider alternating LC series circuit consisting of a linear inductor and a capacitor of two terminals as a dipole as shown in Figure 1. Also, it is known that a functional relationship between the electric charge $q$, the capacitor voltage $u \equiv u(q)$, and the time charging $t$ has the form as follows:

$$
f(q, u, t)=0 .
$$

The relationship between the charge $q$ of the nonlinear capacitor and the voltage drop across it may be approximated by the following quadratic equation [29]:

$$
u_{c}=s q+a q^{2}
$$

where $u_{c}$ gives the potential across the plates of the nonlinear capacitor and $s$ and $a$ are constants related to the capacitor.

Now, Kirchhoff's voltage law for the LC series circuit could be written as follows:

$$
L i^{\prime}+s q+a q^{2}=V_{E}
$$

where $i^{\prime} \equiv \partial_{t} i, L$ is the inductor inductance measured by Henry unit, and $V_{E}$ gives the voltage of the battery which is constant. The relation between the current and charge reads $i=\mathrm{d} q / \mathrm{d} t$, and by rearrange equation (4), the Helmholtz equation is obtained as

$$
\ddot{q}+\alpha+\beta q+\gamma q^{2}=0
$$

where $q \equiv q(t), \ddot{q} \equiv \partial_{t}^{2} q, \beta=s / L=1 /(\mathrm{LC})=\omega_{0}^{2}$, where $\omega_{0}=$ $1 / \sqrt{\mathrm{LC}}$ represents the resonant angular frequency, and $\gamma=a / L=1 /\left(C q_{0} L\right)$, where $C$ donates the capacitance of the capacitor in farad and $q_{0}$ represent the initial value of charge on the capacitor plates which has minimum or zero value for the charging capacitor and for discharge, it has maximum values. The LC circuit is considered as an oscillating circuit which stores energy to oscillate at the resonant frequency $f_{0}=1 /(2 \pi \sqrt{\mathrm{LC}})$ of the circuit where $f_{0}$ could be calculated from the resonance condition: inductor impedance $\left(2 \pi f_{0} L\right)=$ capacitor impedance $\left(1 /\left(2 \pi f_{0} C\right)\right)$.

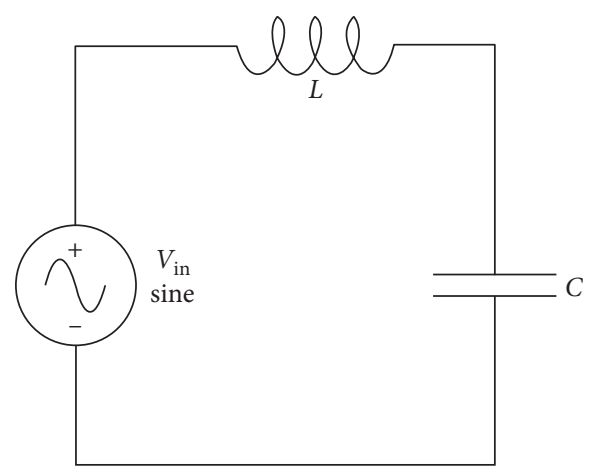

FIgURE 1: The LC series circuit.

2.2. Electronegative Plasmas and the KdV-Helmholtz Equation. Let us consider the propagation of electrostatic nonlinear ion-acoustic structures in a collisionless electronegative plasma consisting of thermal particles (including Maxwellian electrons and light negative ions) and fluid cold positive ions. The dynamics of nonlinear electrostatic structures are governed by the following dimensionless fluid equations [30-33]:

$$
\begin{gathered}
\partial_{t} n+\partial_{x}(n u)=0, \\
\partial_{t} u+u \partial_{x} u+\partial_{x} \phi=0, \\
\partial_{x}^{2} \phi=\mu_{e} \exp [\phi]+\mu_{n} \exp \left[\sigma_{n} \phi\right]-n,
\end{gathered}
$$

where $n$ and $u$ are the normalized positive ion number density and fluid velocity, respectively, and $\phi$ gives the normalized electrostatic potential [30]. Here, $\sigma_{n}=T_{e} / T_{n}$ gives the temperature ratio of electron-to-negative ion, $\mu_{e}=n_{e}^{(0)} / n^{(0)}$ gives the electron concentration, and $\mu_{n}=n_{n}^{(0)} / n^{(0)}$ refers to the negative concentration, where $n^{(0)}, n_{e}^{(0)}$, and $n_{n}^{(0)}$ are the unperturbed equilibrium number densities of the positive ion, electron, and negative ion, respectively. Accordingly, the neutrality condition at equilibrium reads $\mu_{e}+\mu_{n}=1$, with $\mu_{n}=\alpha_{n} /\left(1+\alpha_{n}\right)$ and $\mu_{e}=1 /\left(1+\alpha_{n}\right)$, where $\alpha_{n}=n_{n}^{(0)} / n_{e}^{(0)}$.

By applying the RPT, the independent variables are given by $\zeta=\varepsilon^{1 / 2}\left(x-V_{\mathrm{ph}} t\right)$ and $\tau=\varepsilon^{3 / 2} t$ and the dependent quantities are expanded as $n=1+\varepsilon n^{(1)}+\varepsilon^{2} n^{(2)}+\cdots$, $u=\varepsilon u^{(1)}+\varepsilon^{2} u^{(2)}+\cdots$, and $\phi=\varepsilon \phi^{(1)}+\varepsilon^{2} \phi^{(2)}+\cdots$. Here, $V_{\mathrm{ph}}$ is the normalized wave phase velocity and $\varepsilon$ is a real and small parameter $(\varepsilon \ll 1)$. Inserting stretching and expansion values of the independent and dependent quantities into equations (6)-(8), we get a system of reduced equations with different orders of $\varepsilon$. The first-order dependent quantities $\left(n^{(1)}, u^{(1)}, \phi^{(1)}\right)$ could be obtained from solving the system of the lowest orders in $\varepsilon$ as $n^{(1)}=\phi^{(1)} / V_{\mathrm{ph}}^{2}, u^{(1)}=\phi^{(1)} / V_{\mathrm{ph}}$, and $V_{\mathrm{ph}}=\sqrt{1 / S_{1}}$. By solving the system of reduced equations to the next order in $\varepsilon$, the following $\mathrm{KdV}$ equation is obtained:

$$
\partial_{\tau} \varphi+K_{1} \varphi \partial_{\zeta} \varphi+K_{2} \partial_{\zeta}^{3} \varphi=0
$$

where the coefficient of the nonlinear term $K_{1}=V_{\mathrm{ph}}^{3}\left(3 / V_{\mathrm{ph}}^{4}-2 S_{2}\right) / 2$ and the coefficient of the dispersion term $K_{2}=V_{\mathrm{ph}}^{3} / 2$, where $S_{1}=\left(\mu_{e}+\mu_{n} \sigma\right)$ and 
$S_{2}=\left(\mu_{e}+\mu_{n} \sigma^{2}\right) / 2$. Note that, for $K_{1}>0\left(K_{1}<0\right)$, the positive (negative) pulses can exist and propagate in the present plasma model. The sign and the values of $K_{1}$ are related to the relevant plasma parameters.

Using the travelling wave transform $\xi=(\zeta+\lambda \tau)$ and $\varphi(\zeta, \tau)=q(\xi)$ into equation (9) and integrating the obtained result over $\xi$, we get

$$
q^{\prime \prime}+\alpha+\beta q+\gamma q^{2}=0
$$

where $q \equiv q(\xi), \quad \dot{q} \equiv \partial_{\xi} q, \quad \ddot{q} \equiv \partial_{\xi}^{2} q$, and $\lambda$ represents the normalized velocity of the moving frame. Here, $\alpha=c_{k} / K_{2}$, where $c_{k}$ is the integrating constant, $\beta=\lambda / K_{2}$, and $\gamma=K_{1} /\left(2 K_{2}\right)$

$$
q^{\prime \prime}+\alpha q+\beta q^{2}=0
$$

\section{Exact Solution}

In this section, we are going to solve, in general, the equation:

$$
\begin{aligned}
\ddot{q}+\alpha+\beta q+\gamma q^{2} & =0, \\
q(0) & =q_{0}, \\
q^{\prime}(0) & =\dot{q}_{0} .
\end{aligned}
$$

To do that, the following ansatz is suggested:

$$
q(t)=A+\frac{B}{1+C \wp\left(t-t_{0} ; g_{2}, g_{3}\right)},
$$

where $B C \neq 0, \wp(t)$ stands for the Weierstrass elliptic function. This function satisfies the following ordinary differential equation (ODE):

$$
\begin{aligned}
& \wp^{\prime}\left(\mathscr{F} ; g_{2}, g_{3}\right)^{2}=4 \wp^{3}\left(\mathscr{F} ; g_{2}, g_{3}\right)-g_{1} \wp\left(\mathscr{F} ; g_{2}, g_{3}\right)-g_{3}, \\
& \wp^{\prime \prime}\left(\mathscr{F} ; g_{2}, g_{3}\right)=-\frac{g_{2}}{2}+6 \wp^{2}\left(\mathscr{F} ; g_{2}, g_{3}\right) .
\end{aligned}
$$

Inserting ansatz (13) into (12) and making use of (15) gives $R(t)=0$, where

$$
\begin{aligned}
R(t)= & C^{2}\left(A^{2} C \gamma+A C \beta+2 B+C \alpha\right) \wp^{3}\left(t-t_{0} ; g_{2}, g_{3}\right) \\
& +C\left(3 A^{2} C \gamma+2 A B C \gamma+3 A C \beta+B C \beta-6 B+3 C \alpha\right) \\
& \wp^{2}\left(t-t_{0} ; g_{2}, g_{3}\right) \\
& +\frac{1}{2} C\left(6 A^{2} \gamma+8 A B \gamma+6 A \beta+2 B^{2} \gamma-3 B C g_{2}\right. \\
& +4 B \beta+6 \alpha) \wp\left(t-t_{0} ; g_{2}, g_{3}\right) \\
& +A^{2} \gamma+2 A B \gamma+A \beta+B^{2} \gamma-2 B C^{2} g_{3}+\frac{1}{2} B C g_{2}+B \beta+\alpha .
\end{aligned}
$$

Equating the coefficients of $\wp^{j}\left(t-t_{0} ; g_{2}, g_{3}\right)$ to zero gives an algebraic system, and by solving it, we obtain

$$
\begin{aligned}
& B=-\frac{6}{2 A \gamma+\beta}\left(A^{2} \gamma+A \beta+\alpha\right) \\
& C=\frac{12}{2 A \gamma+\beta}, \\
& g_{2}=\frac{1}{12}\left(\beta^{2}-4 \alpha \gamma\right), \\
& g_{3}=\frac{1}{216}(2 A \gamma+\beta)\left(-2 A^{2} \gamma^{2}-2 A \beta \gamma-6 \alpha \gamma+\beta^{2}\right),
\end{aligned}
$$

and thus, we get

$$
q(t)=A-\frac{6(A(A \gamma+\beta)+\alpha)}{12 \gamma\left(t-t_{0} ;(1 / 12)\left(\beta^{2}-4 \alpha \gamma\right),(1 / 216)(\beta+2 A \gamma)\left(\beta^{2}-2 A \gamma \beta-2 \gamma\left(\gamma A^{2}+3 \alpha\right)\right)\right)+2 A \gamma+\beta} .
$$

Now, for determining the values of $t_{0}$ and $A$, the initial conditions $\left(q(0)=q_{0}\right.$ and $\left.q^{\prime}(0)=\dot{q}_{0}\right)$ must be applied in addition to the following formula:

$$
\begin{aligned}
\wp\left(z+y ; g_{2}, g_{3}\right)= & \frac{1}{4}\left(\frac{\wp^{\prime}\left(z ; g_{2}, g_{3}\right)-\wp^{\prime}\left(y ; g_{2}, g_{3}\right)}{\wp\left(z ; g_{2}, g_{3}\right)-\wp\left(y ; g_{2}, g_{3}\right)}\right)^{2} \\
& -\wp\left(z ; g_{2}, g_{3}\right)-\wp\left(y ; g_{2}, g_{3}\right),
\end{aligned}
$$

where $z=t$ and $y=-t_{0}$ for our case. According to this relation, we get

$$
\wp\left(t-t_{0} ; g_{2}, g_{3}\right)=\frac{1}{4}\left(\frac{\wp^{\prime}\left(t ; g_{2}, g_{3}\right)-c_{1}}{\wp\left(t ; g_{2}, g_{3}\right)-c_{0}}\right)^{2}-\wp\left(t ; g_{2}, g_{3}\right)-c_{0},
$$

where $c_{0}=\wp\left(-t_{0} ; g_{2}, g_{3}\right)$ and $c_{1}=\wp^{\prime}\left(-t_{0} ; g_{2}, g_{3}\right)$.

The initial conditions $\left(q(0)=q_{0}\right.$ and $\left.q^{\prime}(0)=\dot{q}_{0}\right)$ give

$$
\begin{aligned}
& c_{0}=-\frac{-q_{0}(2 A+B)+A(A+B)+B \dot{q}_{0}+q_{0}^{2}}{C\left(A-q_{0}\right)^{2}}, \\
& c_{1}=-\frac{B \dot{q}_{0}}{C\left(A-q_{0}\right)^{2}},
\end{aligned}
$$

where the values of $B$ and $C$ are defined in equation (17). 
Finally, the value of $A$ can be estimated from the condition

$$
\ddot{q}(0)+\alpha+\beta q(0)+\gamma q^{2}(0)=0
$$

which gives

$$
\frac{2\left(2 \gamma A^{3}+3 \beta A^{2}+6 \alpha A-6 \alpha q_{0}-3 \beta q_{0}^{2}-2 \gamma q_{0}^{3}-3 q_{0}^{2}\right)}{3\left(A-q_{0}\right)}=0 .
$$

It is clear that $A$ must obey the cubic equation:

$$
\gamma A^{3}+3 \beta A^{2}+6 \alpha A-\left(6 \alpha q_{0}+3 \beta q_{0}^{2}+2 \gamma q_{0}^{3}+3 q_{0}^{2}\right)=0 .
$$

We will choose the first root of equation (24), i.e.,

$$
A=\frac{1}{2 \gamma}\left(\begin{array}{c}
\sqrt[3]{\frac{\Delta}{27}+6 \gamma^{2}\left(2 \alpha q_{0}+\beta q_{0}^{2}+q_{0}^{2}\right)+6 \alpha \beta \gamma-\beta^{3}+4 \gamma^{3} q_{0}^{3}} \\
+\frac{\beta^{2}-4 \alpha \gamma}{\sqrt[3]{(\Delta / 27)+6 \gamma^{2}\left(2 \alpha q_{0}+\beta q_{0}^{2}+q_{0}^{2}\right)+6 \alpha \beta \gamma-\beta^{3}+4 \gamma^{3} q_{0}^{3}}}-\beta
\end{array}\right),
$$

with

$$
\Delta=\sqrt{729\left(\left(\beta+2 \gamma q_{0}\right)\left(-6 \alpha \gamma+\beta^{2}-2 \gamma q_{0}\left(\beta+\gamma q_{0}\right)\right)-6 \gamma^{2} q_{0}^{2}\right)^{2}+\left(36 \alpha \gamma-9 \beta^{2}\right)^{3}}
$$

For $\dot{q}_{0}=0$, i.e., $q(0)=q_{0}$ and $q^{\prime}(0)=0$, the following solution is obtained:

$$
q(t)=q_{0}-\frac{6\left(\alpha+\beta q_{0}+\gamma q_{0}^{2}\right)}{\beta+2 \gamma q_{0}+12 \wp\left(q ;(1 / 12)\left(\beta^{2}-4 \alpha \gamma\right),(1 / 216)\left(\beta+2 \gamma q_{0}\right)\left(\beta^{2}-2 \gamma q_{0} \beta-2 \gamma^{2} q_{0}^{2}-6 \alpha \gamma\right)\right)} .
$$

In the case, when $\dot{q}_{0} \neq 0$, the solution to initial value problem (1) is given by

$$
q(t)=A+\frac{B}{1+C\left((1 / 4)\left(\left(c_{1}-\wp^{\prime}\left(t ; g_{2}, g_{3}\right)\right) /\left(c_{0}-\wp\left(t ; g_{2}, g_{3}\right)\right)\right)^{2}-c_{0}-c_{1}-\wp\left(t ; g_{2}, g_{3}\right)\right)}
$$

or, in a more compact form,

$$
q(t)=A+\frac{B}{1+C \wp\left(t-t_{0} ; g_{2}, g_{3}\right)}
$$

where $t_{0}=-\wp^{-1}\left(c_{0} ; g_{2}, g_{3}\right)$.

Expression $\wp^{-1}\left(c_{0} ; g_{2}, g_{3}\right)$ stands for the inverse of the Weierstrass elliptic function which is defined as

$$
\wp^{-1}\left(x ; g_{2}, g_{3}\right)=\int_{\infty}^{x} \frac{1}{\sqrt{4 \zeta^{3}-g_{2} \zeta-g_{3}}} \mathrm{~d} \zeta
$$

for real $x$ and $\mathfrak{R e}\left(4 \zeta^{3}-g_{2} \zeta-g_{3}\right)>0$.
The obtained solution (29) is periodic with period

$$
T= \pm 2 \int_{e_{1}}^{\infty} \frac{1}{\sqrt{4 z^{3}-g_{2} z-g_{3}}} \mathrm{~d} z,
$$

where $e_{1}$ is the greatest real root of the cubic

$$
4 z^{3}-g_{2} z-g_{3}=0 \text {. }
$$

Taking (30) into account, the period $T$ in (29) may also be expressed as

$$
T= \pm 2 \wp^{-1}\left(e_{1} ; g_{2}, g_{3}\right) .
$$

Remark 1. The Weierstrass elliptic function $\wp\left(z ; g_{2}, g_{3}\right)$ is related to the Jacobian elliptic functions sn, $c d$, and dn as follows: 


$$
\begin{aligned}
\operatorname{dn}(\sqrt{\omega} z m)^{2} & =1-\frac{3 m \omega}{3 \wp\left(z ;(4 / 3)\left(m^{2}-m+1\right) \omega^{2},(4 / 27)(m-2)(m+1)(2 m-1) \omega^{3}\right)}, \\
\wp\left(z ; g_{2}, g_{3}\right) & =-\frac{m \omega}{\operatorname{dn}(\sqrt{\omega} z \mid m)^{2}-1}-\frac{1}{3}(m+1) \omega, \\
& =\frac{\omega}{\operatorname{sn}(\sqrt{\omega} z \mid m)^{2}}-\frac{1}{3}(m+1) \omega, \\
& =\frac{\omega}{1-\operatorname{cn}(\sqrt{\omega} z \mid m)^{2}}-\frac{1}{3}(m+1) \omega,
\end{aligned}
$$

where

$$
\begin{gathered}
16 \omega^{6}-24 \omega^{4} g_{2}+9 \omega^{2} g_{2}^{2}-g_{2}^{3}+27 g_{3}^{2}=0 \\
4\left(g_{2}^{3}-27 g_{3}^{2}\right) \omega^{6}-12\left(g_{2}^{3}-27 g_{3}^{2}\right) \omega^{5}-3\left(g_{2}^{3}+216 g_{3}^{2}\right) \omega^{4} \\
+2\left(13 g_{2}^{3}+378 g_{3}^{2}\right) \omega^{3} \\
-3\left(g_{2}^{3}+216 g_{3}^{2}\right) \omega^{2}-12\left(g_{2}^{3}-27 g_{3}^{2}\right) \omega+4 g_{2}^{3}-108 g_{3}^{2}=0
\end{gathered}
$$

In view of (35), the fundamental period of the Weierstrass function $\wp\left(z ; g_{2}, g_{3}\right)$ may also be expressed in the following form:

$$
T=\frac{2 K(m)}{\sqrt{\omega}} .
$$

Further properties of the Weierstrass function with applications in quantum theory of Lame potential may be found in [26].

\section{Results and Discussions}

Let us consider a nonlinear $L C$ series circuit with $L=1 H$, $C=0.1 F, q_{o}=1 C$, and let the circuit without external battery, i.e., $V_{E}=0(\alpha=0)$. Thus, the exact solution to the initial value problem

$$
\begin{aligned}
& \ddot{q}+10 q+q^{2}=0, \\
& q(0)=q_{0}=1, \\
& q^{\prime}(0)=\dot{q}_{0}=0,
\end{aligned}
$$

according to formula (27), is given by

$$
q(t)=1-\frac{11}{2(1+\wp(t ;(25 / 3),(13 / 3)))} .
$$

This solution is periodic, and to find its period according to equation (32), the following cubic equation is solved:

$$
4 z^{3}-\frac{25}{3} z-\frac{13}{3}=0,
$$

which is given the following three real roots:

$$
z_{1}=-1
$$$$
z_{2}=\frac{3+4 \sqrt{3}}{6}
$$

$$
z_{3}=\frac{3-4 \sqrt{3}}{6}
$$

The greatest root is $e_{1}=z_{2}$.

Using formula (31), we obtain

$$
\begin{aligned}
T & =\int_{z_{2}}^{\infty} \frac{1}{\sqrt{4 z^{3}-(25 / 3) z-(13 / 3)}} \mathrm{d} z, \\
& =2 \sqrt{\frac{2}{11}(9+4 \sqrt{3})} \mathfrak{R} e\left(K\left(\frac{1}{11}(43+24 \sqrt{3})\right)\right) .
\end{aligned}
$$

Also, we can use equations (34)-(38) to obtain

$$
\begin{aligned}
q(t)= & -4(2+\sqrt{3})+(9+4 \sqrt{3}) \mathrm{dn} \\
& \left(\sqrt{\frac{3}{2}+\frac{2}{\sqrt{3}}} t \mid \frac{1}{11}(43-24 \sqrt{3})\right)^{2},
\end{aligned}
$$

and then, the period of the solution is given by

$$
T=\frac{2 K((1 / 11)(43-24 \sqrt{3}))}{\sqrt{(3 / 2)+(2 / \sqrt{3})}} .
$$

From equation (43) and (45), the period $T$ is obtained as

$$
\begin{aligned}
T & =2 \sqrt{\frac{2}{11}(9+4 \sqrt{3})} \mathfrak{R} e\left(K\left(\frac{1}{11}(43+24 \sqrt{3})\right)\right), \\
& =\frac{2 K((1 / 11)(43-24 \sqrt{3}))}{\sqrt{(3 / 2)+(2 / \sqrt{3})}}, \\
& \approx 1.99590288250676993 .
\end{aligned}
$$

In Figure 2, the analytical solution (40) is compared with the Runge-Kutta (RK) numerical solution, and the agreement between the two solutions is found to be very good. If the initial current value of the LC circuit is not equal to zero, i.e, $i=q^{\prime}(0)=\dot{q}_{0} \neq 0$, but has a specified value $\dot{q}_{0}=0.3 \mathrm{~A}$, in this case, we can consider solution (28) or (29) instead of the first one (27). It is observed that the results obtained by the 


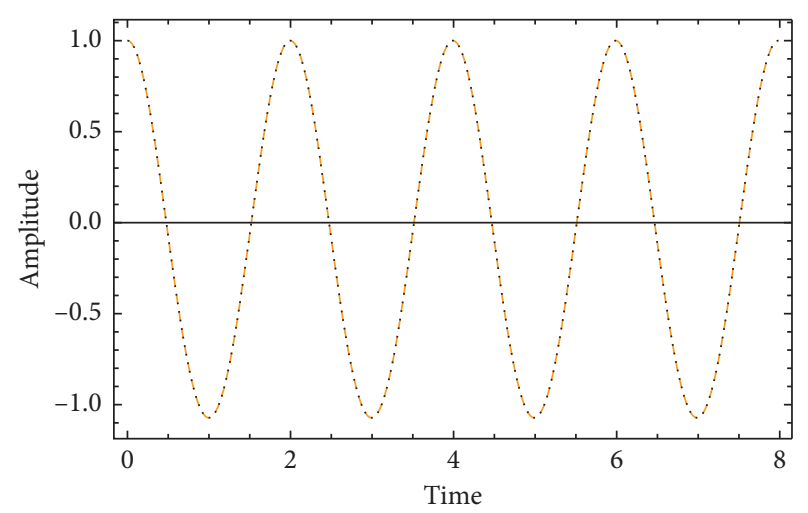

FIgURE 2: (Color online). A comparison between the analytical solution (40) (dashed curve) and the Runge-Kutta (RK) numerical solution (dotted curve) for the LC series circuit with $L=1 H$, $C=0.1 F, q_{0}=1 C$, and $\dot{q}_{0}=0$.

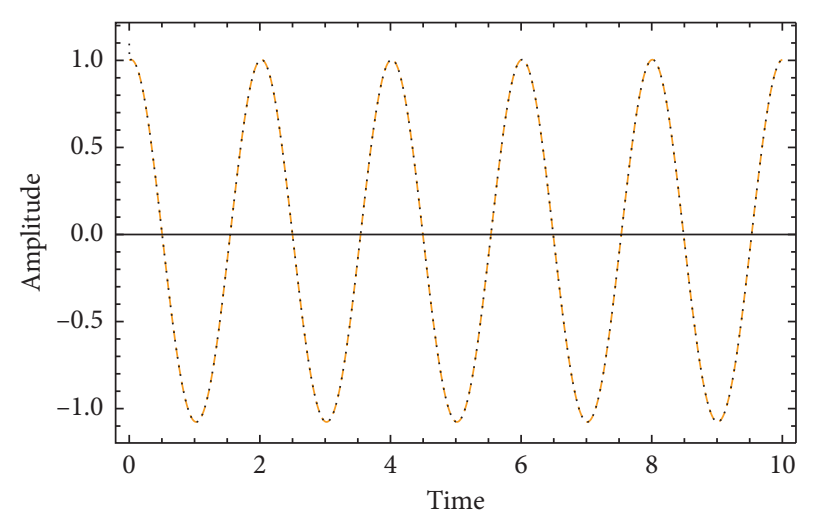

Figure 3: (Color online). A comparison between the analytical solution (28) (dashed curve) and the Runge-Kutta (RK) numerical solution (dotted curve) for the LC series circuit with $L=1 H$, $C=0.1 F, q_{0}=1$, and $\dot{q}_{0}=0.3 \mathrm{~A}$.

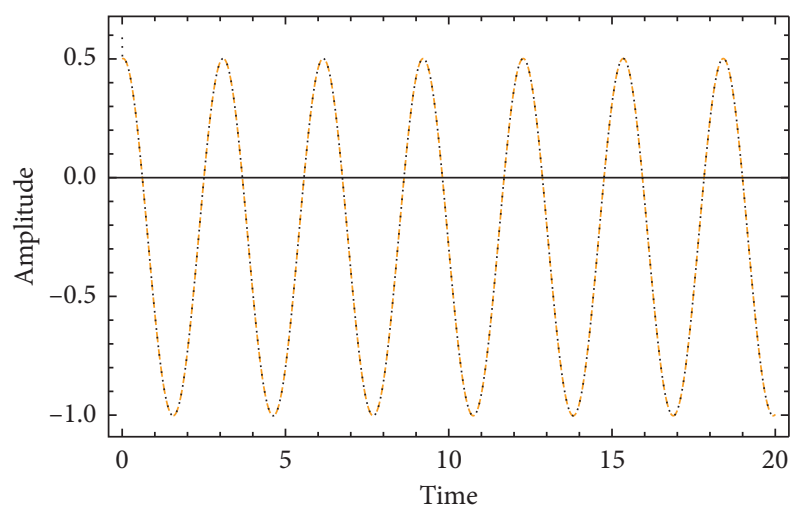

FIgURE 4: (Color online). A comparison between the analytical solution (28) (dashed curve) and the Runge-Kutta (RK) numerical solution (dotted curve) for the electronegative plasma data $\alpha_{n}=0.1, \sigma_{n}=8.7, q_{0}=0.51$, and $\dot{q}_{0}=0.1$.

exact solution are in an excellent agreement with the RK numerical solution, as shown in Figure 3. For the plasma application, our analyses are based on the experimental observations data where electron and negative ion temperatures are, respectively, given by $T_{e} \approx 0.69 \mathrm{eV}$ and $T_{n} \approx(0.06 \pm 0.02) \mathrm{eV}$ and $n_{e}=3.8 \times 10^{9} \mathrm{~cm}^{-3}$ gives the electron number density. According to these data, we get $\sigma_{n}=8.7,11.5$, and 17.25 and the negative ions concentration $0 \leq \mu_{n} \leq 1 \quad[26,27,30]$. Using these data, we get $(\beta, \gamma)=(4.433,0.44)$, and by plotting these data according to solution (27), we note, from Figure 4, the excellent matching between our solution and the RK numerical solution.

\section{Conclusions}

The quadratic nonlinear Helmholtz differential equation is solved analytically for any arbitrary initial conditions through the Weierstrass elliptic function. Accordingly, some new analytical solutions are obtained for the first time using ansatz (12). The proposed methodology is of great importance in solving several physics problems in plasma physics and electronic circuits. For instance, our obtained solutions are devoted for describing the dynamics of nonlinear oscillations that propagate in electronegative complex plasmas. Moreover, our solutions are applied for studying the characteristic behavior of signal oscillations in the LC series circuits.

\section{Data Availability}

No data were used to support this research.

\section{Conflicts of Interest}

The authors declare that they have no conflicts of interest.

\section{Acknowledgments}

The authors thank the University Francisco Jose de Caldas for the support to carry out this work. Also, the authors thank Associate Prof. S.A. El-Tantawy, the chief of Research Center for Physics (RCP), Faculty of Science and Arts, AlBaha University, Saudi Arabia, and Faculty of Science, Port Said University, Egypt, for fruitful discussions, careful reading, and improving their manuscript very well.

\section{References}

[1] A. G. Greenhill, The Applications of Elliptic Functions, Macmillan, London, UK, 1892.

[2] T. O'Neil, "Collisionless damping of nonlinear plasma oscillations," Physics of Fluids, vol. 8, pp. 2255-2262, 1965.

[3] P. G. D. Barkham and A. C. Soudack, "An extension to the method of Kryloff and Bogoliuboff $†$," International Journal of Control, vol. 10, no. 4, pp. 377-392, 1969.

[4] I. F. Collins, "On the theory of rigid/perfectly plastic plates under uniformly distributed loads," Acta Mechanica, vol. 18, no. 3-4, pp. 233-254, 1973.

[5] B. M. Karmakar, "Nonlinear vibrations of orthotropic plates carrying concentrated mass," Journal of Engineering for Industry, vol. 100, no. 2, pp. 293-294, 1978.

[6] J. Zajaczkowski, "Destabilizing effect of Coulomb friction on vibration of a beam supported at an axially oscillating mount," 
Journal of Sound and Vibration, vol. 79, no. 4, pp. 575-580, 1981.

[7] S. S. Chang, "The general solutions of the doubly periodic cracks," Engineering Fracture Mechanics, vol. 18, no. 4, pp. 887-893, 1983.

[8] D. Grozev, A. Shivarova, and A. D. Boardman, "Envelope solitons of surface waves in a plasma column," Journal of Plasma Physics, vol. 38, no. 3, pp. 427-437, 1987.

[9] A. I. Manevich, "Interaction of coupled modes accompanying non-linear flexural vibrations of a circular ring," Journal of Applied Mathematics and Mechanics, vol. 58, no. 6, pp. 1061-1068, 1994.

[10] R. H. Rand, R. J. Kinsey, and D. L. Mingori, "Dynamics of spinup through resonance," International Journal of NonLinear Mechanics, vol. 27, no. 3, pp. 489-502, 1992.

[11] W. Hu and D. J. Scheeres, "Spacecraft motion about slowly rotating asteroids," Advances in the Astronautical Sciences, vol. 105, pp. 839-848, 2000.

[12] W. Lestari and S. Hanagud, "Nonlinear vibration of buckled beams: some exact solutions," International Journal of Solids and Structures, vol. 38, no. 26-27, pp. 4741-4757, 2001.

[13] S. Liu, Z. Fu, S. Liu, and Q. Zhao, "Jacobi elliptic function expansion method and periodic wave solutions of nonlinear wave equations," Physics Letters A, vol. 289, no. 1-2, pp. 69-74, 2001.

[14] J. L. Hammack and D. M. Henderson, "Experiments on deepwater waves with two-dimensional surface patterns," Journal of Offshore Mechanics and Arctic Engineering, vol. 125, no. 1, pp. 48-53, 2003.

[15] V. S. Aslanov, "The oscillations of a body with an orbital tethered system," Journal of Applied Mathematics and Mechanics, vol. 71, no. 6, pp. 926-932, 2007.

[16] A. H. Salas and J. E. Castillo, "Exact solutions for a nonlinear model," Applied Mathematics and Computations, vol. 217, no. 4, pp. 1646-1651, 2010.

[17] A. H. Salas and J. E. Castillo, "La ecuación Seno-Gordon perturbada en la Dinámica no lineal del ADN," Revista Mexicana de Fisica, vol. 58, pp. 481-487, 2012.

[18] P. J. Jolmes, "A nonlinear oscillator with a strange attractor," Proceedings of the Royal Society, vol. 292, pp. 419-448, 1979.

[19] S. A. Khuri and S. Xie, "On the numerical verification of the asymptotic expansion of duffing's equation," International Journal of Computer Mathematics, vol. 72, no. 3, pp. 325-330, 1999.

[20] F. Mirzaee and N. Samadyar, "Combination of nite difference method and meshless method based on radial basis functions to solve fractional stochastic advection diffusion equations," Engineering with Computers, 2019.

[21] N. Samadyar and F. Mirzaee, "Numerical solution of twodimensional weakly singular stochastic integral equations on non-rectangular domains via radial basis functions," Engineering Analysis with Boundary Elements, vol. 101, pp. 27-36, 2019.

[22] F. Mirzaee and N. Samadyar, "Numerical solution based on two-dimensional orthonormal Bernstein polynomials for solving some classes of two-dimensional nonlinear integral equations of fractional order," Applied Mathematics and Computation, vol. 344-345, pp. 191-203, 2019.

[23] Y. Liu and G.-R. Li, "Matter wave soliton solutions of the cubic-quintic nonlinear Schrödinger equation with an anharmonic potential," Applied Mathematics and Computation, vol. 219, no. 9, pp. 4847-4852, 2013.

[24] Y. Geng, J. Li, and L. Zhang, "Exact explicit traveling wave solutions for two nonlinear Schrödinger type equations,"
Applied Mathematics and Computation, vol. 217, no. 4, pp. 1509-1521, 2010.

[25] N. Nayfeth and D. T. Mook, Non-linear Oscillations, John Wiley, New York, NY, USA, 1973.

[26] J. A. Almendral and M. A. F. Sanjun, "Integrability and symmetries for the Helmholtz oscillator with friction," Journal of Physics A: Mathematical and General, vol. 36, no. 3, p. $695,2003$.

[27] S. Morfa and J. C. Comte, "A nonlinear oscilators netwok devoted to image processing," International Journal of Bifurcation and Chaos, vol. 14, no. 4, pp. 1385-1394, 2009.

[28] A. H. Salas and J. E. Castillo, "Exact solutions to cubic Duffing equation for a nonlinear electrical circuit," Visión ElectrónicaAlgo más que un estado sólido, vol. 8, no. 1, 2014.

[29] E. Gluskin, "A nonlinear resistor and nonlinear inductor using a nonlinear capacitor," Journal of the Franklin Institute, vol. 336, no. 7, pp. 1035-1047, 1999.

[30] S. A. El-Tantawy, "Nonlinear dynamics of soliton collisions in electronegative plasmas: the phase shifts of the planar KdVand mkdV-soliton collisions," Chaos, Solitons \& Fractals, vol. 93, pp. 162-168, 2016.

[31] S. A. El-Tantawy and T. Aboelenen, "Simulation study of planar and nonplanar super rogue waves in an electronegative plasma: local discontinuous Galerkin method," Physics of Plasmas, vol. 24, Article ID 052118, 2017.

[32] S. A. El-Tantawy, A. M. Wazwaz, and S. Ali Shan, "On the nonlinear dynamics of breathers waves in electronegative plasmas with Maxwellian negative ions," Physics of Plasmas, vol. 24, Article ID 022105, 2017.

[33] S. A. El-Tantawy, "Tarek Aboelenen, and Sherif M E Ismaeel, local discontinuous Galerkin method for modeling the nonplanar structures (solitons and shocks) in an electronegative plasma," Physics of Plasmas, vol. 26, Article ID $022115,2019$. 